\title{
OBSERVATORIO
}

\section{IRC 2.0. MEDIOS PARA LA INFORMACIÓN, LA RELACIÓN Y LA COMUNICACIÓN EN LA WEB 2.0}

\section{Antonio Fumero-Reverón}

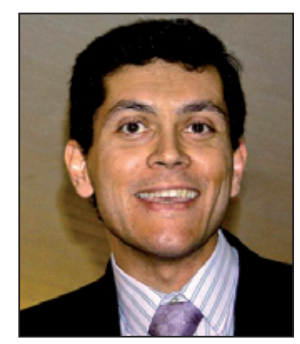

Antonio Fumero-Reverón es ingeniero de telecomunicación y MBA por la Universidad Politécnica de Madrid (UPM). Especialista en la gestión de la tecnología e innovación, comenzó su carrera profesional en la Unidad de Desarrollo Tecnológico e Innovación de BBVA. Actualmente desempeña su actividad como investigador en la UPM, colaborando con la Cátedra Telefónica para Internet de Nueva Generación y el Grupo de Tecnologías de la Información para la Gestión Empresarial. Es socio fundador de Win-Win Consultores, y participa en otras iniciativas empresariales dentro del mismo ámbito, como TalentBrokers. Es coautor del libro Web 2.0 (2007).

\section{Universidad Politécnica de Madrid ETSI de Telecomunicación, Despacho A-126 Av. Complutense, 30. 28040 Madrid amfumero@gmail.com}

\section{Resumen}

El ritmo acelerado al que llegan y desaparecen tendencias, novedades, herramientas, tecnologías, dispositivos, servicios, etc., en nuestra sociedad de la información exige a cualquier ciudadano cierta capacidad para interiorizar esa complejidad. En el artículo se presenta y desarrolla muy brevemente un modelo simplificado que nos ayudará en el análisis de tres fenómenos clave para entender el estadio evolutivo actual de la Web como parte más visible de la Red: el periodismo ciudadano, las redes sociales y la Web en tiempo real. Se basa en tres elementos (infotecnologías, personas y contenidos) y se articula sobre tres ejes (información, relación y comunicación). El modelo quiere servir al lector como herramienta intelectual y referencia conceptual para profundizar en cualquiera de sus dimensiones.

\section{Palabras clave}

Web 2.0, Medios sociales, Redes sociales, Comunicación, Información, Relación, Web en tiempo real, Facebook, Twitter.

\begin{abstract}
The rapid pace at which trends, news, tools, technologies, devices, services, etc. come and go in our information society requires of every citizen a certain capacity to internalize the perceived complexity behind the metaphors we are used to hearing from IT professionals, as well as to assess the impact of these changes on everyday life. The article briefly introduces and develops a simplified model that helps in presenting three key phenomena that are relevant enough for understanding the current developmental stage of the Web as most visible part of the Network itself. It is based on three elements (infotechnologies, people and content) and it is developed along three axes (information, relations and communication). The model also wants to serve the reader as an intellectual and conceptual tool to delve into any of its dimensions.
\end{abstract}

\section{Keywords}

Web 2.0, Social networks, Social media, Communication, Information, Relationships, Real time Web, Facebook, Twitter.

Fumero-Reverón, Antonio. "IRC 2.0. Medios para la información, la relación y la comunicación en la web 2.0". El profesional de la información, 2011, noviembre-diciembre, v. 20, n. 6, pp. 605-609.

http://dx.doi.org/10.3145/epi.2011.nov.01

\section{Introducción}

IRC (internet relay chat) es un protocolo de comunicación en tiempo real basado en texto, que permite la persistencia de las conversaciones, organizadas en "canales". Creado por Jarkko Oikarinen en la Universidad de Oulu, Finlandia, durante el verano de 1988, sería superado por la amplia popu- larización de los servicios de mensajería instantánea como Gtalk o ICQ, las redes sociales como Facebook y Tuenti, y el fenómeno Twitter; pero constituye la metáfora perfecta de la realidad de una Web en tiempo real (Sainz-Peña, 2011). Una Web que evoluciona a toda velocidad mientras transforma las tres dimensiones básicas de nuestra naturaleza social: la información, la relación y la comunicación, al rit- 
mo que sigue marcando aún hoy la retórica informática del cambio de versión.

En este breve artículo editorial, desarrollaré las características transformadoras que podemos identificar en esos tres ejes íntimamente relacionados, como parte de un modelo. Es una simplificación de lo que en palabras de Fernando Sáez-Vacas constituye un Nuevo entorno tecnosocial (NET): un contexto creciente en complejidad en el que hacemos un uso intensivo, cotidiano, de numerosas herramientas y tecnologías -más o menos "convivenciales" (Illich, 1973)-, que nos transforman mientras avanzamos en un proceso de construcción colectiva que llamamos Sociedad de la información.

\section{La Web evoluciona a toda velocidad} mientras transforma las tres dimensiones básicas de nuestra naturaleza social: información, relación y comunicación

Tres fenómenos sociotécnicos servirán para ilustrar aquí las tres dimensiones en las que quiero estructurar las transformaciones que experimentamos en nuestra sociedad red:

- periodismo ciudadano, que se ha convertido en un fenómeno sustantivo que debemos entender y conocer para enfrentarnos como ciudadanos a un nuevo escenario informativo;

- redes sociales, especificidad más popularizada de los medios sociales (social media), que se han convertido en el lugar común de la práctica totalidad de la actualidad relacionada directamente con la Red;

- Web en tiempo real, cuya instantaneidad y veloz obsolescencia quedan perfectamente ilustradas por el fenómeno Twitter o los servicios de geolocalización socialmente referenciados como Foursquare.

El fenómeno "dos punto cero" se popularizó a partir de los años 2002 a 2004, tras el colapso provocado por la locura financiera asociada a los valores de las empresas tecnológicas que centraban sus actividades en internet. Propugnando un "cambio de versión" al adoptar la retórica informática, ha tenido un profundo impacto transformador, dando lugar a cambios drásticos en diferentes industrias, con ritmos muy distintos.

En España este fenómeno se consolidaba hace apenas un lustro alrededor de los blogs, investidos a principios de siglo de cierto carácter paradigmático como quintaesencia del supuesto impacto "democratizador" de la Red. En un contexto extremadamente complejo crecía la disponibilidad masiva de herramientas, generalmente construidas alrededor del formato blog y/o wiki y algunas tecnologías asociadas, como los gestores de contenidos (CMS, content management systems), los formatos estándar para la sindicación de contenidos, o los microformatos semánticos, "tagsonomías" o folksonomías.

Estas aplicaciones se veían amplificadas funcionalmente por un escenario en el que se disparaba el número, la variedad y la accesibilidad de dispositivos móviles e inalámbricos, además de las conexiones de banda ancha fija y móvil, que se abarataban en gran parte por la competencia salvaje en precios a la que el marco regulador obligaba a las tradicionales telecos.

\section{Facebook juega a reinventar la propia Web a partir de una base relacional y no documental, tal y como sucedía con la primigenia world wide web}

\section{Información, relación y comunicación}

En este escenario la primera industria seriamente impactada es la relacionada directamente con la producción de contenidos, audiovisuales y, por supuesto, informativos. La apropiación social de herramientas y tecnologías se ha hecho más y más evidente por ejemplo en situaciones de conflictos armados o desastres naturales, permitiendo: dar una mayor cobertura a las ayudas internacionales para el terremoto de Haití; apoyar la movilización social en los países del norte de África durante la primavera árabe; articular movimientos de alcance e innegable trascendencia como el \#15M; obtener imágenes de primera mano de los atentados terroristas en Londres; o montar medios hiperlocales en comunidades en desarrollo en Latinoamérica.

Más allá de mejorar la "convivencialidad" de las propias herramientas e (info)tecnologías, el proceso de co-evolución en el que se encuentran sumidas ciudadanía y tecnología, impacta en profesiones como la periodística, introduciendo corrientes como el periodismo de datos y el periodismo informático o computational journalism (Cohen; Hamilton; Turner, 2011). La participación ciudadana se convierte en un recurso muy valioso para una profesión periodística que cambia drásticamente y que se ve amenazada en muchas ocasiones precisamente por esa participación mal entendida y poco articulada. Con todo, el fenómeno del periodismo ciudadano se consolida con el tiempo y empieza a calar en numerosos actores tradicionales, cabeceras conocidas y grupos mediáticos.

\section{Nuestro tiempo en la Red lo ocupan las redes sociales, la nueva ágora en la que reinventamos nuestra actividad social más natural: la relacional}

Nos informamos de manera distinta y navegamos de hecho de forma diferente: nuestro tiempo en la Red lo ocupan las redes sociales, una nueva suerte de ágora en la que reinventamos nuestra actividad social más natural: la relacional. La práctica totalidad de los sitios web que se habían arrogado el adjetivo "dos punto cero" y que permiten a los usuarios editar, publicar y compartir contenidos de todo tipo, ahora utilizan el adjetivo "social", añadiendo una variedad considerable y creciente de funcionalidades relacionales.

Mientras tanto, Facebook -convertida en la nueva Google, que a su vez se ha arrogado el puesto de la ancestral Micro- 
soft-juega a reinventar la propia Web precisamente a partir de una base relacional y no documental, tal y como sucedía con la primigenia world wide web de la que aún hoy somos todos herederos.

Es una situación en la que se han vuelto a revalorizar las propuestas originales de los padres de la Web, que siempre estuvieron construidas sobre la visión de una red de significados que conectara a las personas, no a las máquinas o a los documentos y contenidos que gestionaban.

Tal es así que la extracción de esos significados de entre una profundidad creciente de grandes datos difícilmente traducibles en información utilizable, se ha convertido en una actividad relevante, prioritaria y directamente relacionada con las capacidades asociadas tradicionalmente a los profesionales de la información y que hoy se intentan integrar en un corpus complejo e interdisciplinar, que se desarrolla lentamente bajo el concepto de ciencias de la Web o Web sciences (Hendler et al., 2008).

Twitter es el fenómeno del momento, conocido por algo más de dos terceras partes de los usuarios de internet, aunque utilizado sólo por la sexta parte de ellos (14\%)

Los elementos informativos y relacionales -contenidos y personas- de una red metafóricamente oculta tras la nube informática (cloud computing), son parte sustancial de la actual dinámica acelerada en la que estamos inmersos. La aceleración se produce por una perentoria necesidad de velocidad, de instantaneidad, de disponibilidad en tiempo real, algo que caracteriza el tipo de patrones de comunicación y de herramientas condicionadas por este tipo de uso.

El fenómeno paradigmático, también con nombre propio, que ilustra este particular es Twitter, una herramienta de comunicación asincrónica en formato texto que nació en 2006 y que en el momento de escribir estas líneas certifica la existencia de alrededor de 200 millones de usuarios. Twitter se está convirtiendo de facto en un canal de comunicación web en tiempo real sobre el que se ha construido un sencillo mecanismo relacional del que participa el propio usuario y que está en la base de su éxito empresarial, que la ha situado a la altura de Facebook en términos de popularidad.

\section{Cómo mentir con las estadísticas}

La vertiente más engañosa de este fenómeno transformador -bajo cualquiera de las metáforas que queramos considerar-, es precisamente la que lo ha popularizado, es decir la cuantitativa. Las propias magnitudes que acostumbramos a encontrar en los titulares al uso, habitualmente relacionadas con el número de piezas de contenido audiovisual que los usuarios "suben" a la Red -para dejar constancia, por ejemplo, de la relevancia de lo que se ha dado en llamar user generated content, UGC-, o el número de cuentas que se han dado de alta en determinado servicio para la gestión online de redes sociales, asociado sin pudor alguno con el número de usuarios activos de la red social del mismo nombre, no constituyen otra cosa que un torpe ejercicio para poner en práctica las técnicas de comunicación aprendidas en textos básicos del gremio (Huff, 1954).

Los perfiles de usuario en las redes sociales se pueden considerar como piezas de contenido, susceptibles de ser tratadas de forma mecánica por cualquier algoritmo de recomendación al uso

Podemos encontrarnos con multitud de datos estadísticos (Van-Bellenghem, 2011) que, aunque lejos de la rigurosidad metodológica que podríamos exigir a un trabajo científico, sí corroboran a lo largo de los años ciertas tendencias hacia la consolidación en el uso generalizado de cierto tipo de aplicaciones, como son las redes sociales. En España, resultan de utilidad los datos arrojados por el Observatorio de Redes Sociales de The cocktail analysis para poner en contexto este fenómeno específico de importancia capital para entender la relevancia del modelo propuesto.

Si resumimos los datos estadísticos disponibles, podemos decir -desde un punto de vista cualitativo-, que el fenómeno de las redes sociales está consolidado: cerca de un $90 \%$ de los internautas conocen Facebook y, de hecho, la identifican con la red social. Tuenti se reafirma como el competidor local en España de la red social más conocida a nivel mundial, consiguiendo mayor atención, medida en tiempo de permanencia o conexión de sus usuarios. Mientras tanto, Twitter se muestra como el fenómeno del momento, conocido por algo más de dos terceras partes de los usuarios de internet, aunque utilizado sólo por la sexta parte de ellos (14\%).

Los usuarios habituales de redes sociales se acercan a la utilización simultánea de dos servicios distintos. Ello certifica en cierto sentido la existencia de un fenómeno local de identificación cultural o por patrones de uso/funcionalidad. Se intensifica la presencia de las marcas comerciales en las redes sociales más conocidas, lo cual hace que los estadísticos cuantitativos -basados en el número de cuentas registradas y/o activas asociadas a cada servicio-, sean cada vez menos fiables.

Los informes especializados comienzan a destacar en 2011 cierta saturación de lo social en lo que se refiere a las redes, primando los contenidos (y por tanto las funciones asociadas) a la hora de seleccionar la red principal a la que accedemos. Es una forma de expresar la verticalización o especialización de este tipo de servicios. De hecho, si pensamos en términos de recomendación social y su automatización, los propios perfiles de usuario se pueden considerar como piezas de contenido, susceptibles de ser tratadas de forma mecánica por cualquier algoritmo de recomendación al uso.

\section{Infotecnologías, personas, contenidos}

Para completar el modelo que he glosado en estos párrafos, el lector debe notar que los componentes básicos que 


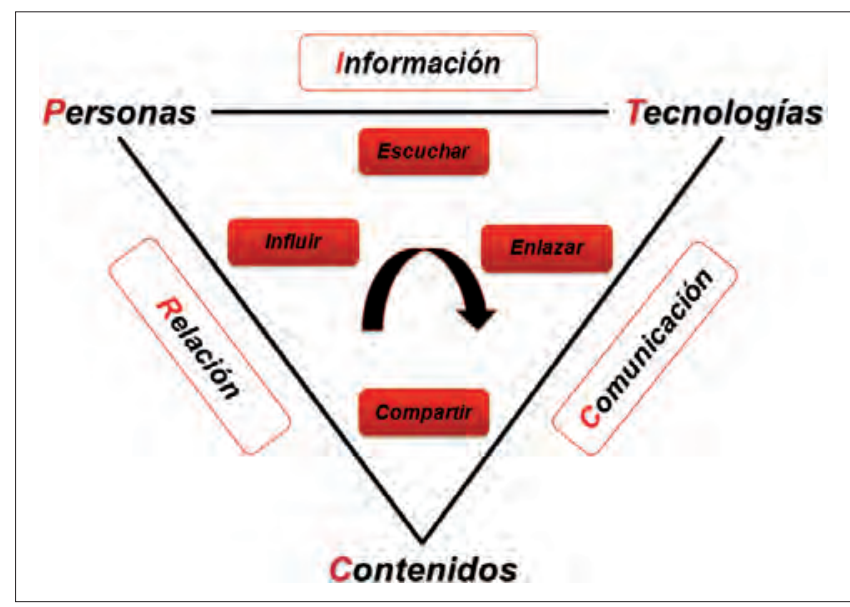

Figura 1. Modelo de aproximación a los medios sociales

intervienen en esas acciones tecnológicamente mediadas -información, relación y comunicación-, son además de las propias infotecnologías, los usuarios (clientes, consumidores, individuos, votantes, ciudadanos, personas...), sujetos socioeconómicos de cualquiera de esas acciones; y los contenidos, datos, piezas informativas, etc., que incorporan cada vez más interacción con esos usuarios (figura 1).

En el escenario que metafóricamente hemos delimitado con esos elementos y los tres ejes que han estructurado este artículo, se desarrollan todo tipo de procesos sociotécnicos en los que toman parte organizaciones empresariales, instituciones y/o individuos a título personal o colectivo:

- Desde el punto de vista político, la emergencia de una nueva ciudadanía (digital) que se apropia de los medios técnicos, ha propiciado movimientos como el \#15M.

- Desde el punto de vista comercial y de marketing, las redes sociales se están convirtiendo en la principal plataforma publicitaria en la Red y fuera de ella.

- Desde el punto de vista sociológico, el incremento que propician tales herramientas en el número y en la intensidad de las relaciones personales hace que se estén redefiniendo de facto conceptos básicos como los de identidad, autoridad o intimidad (privacidad para los anglosajones).

- Desde el ámbito educativo, los paradigmas pedagógicos clásicos se tambalean ante la realidad impuesta por las propias redes y los medios infotecnológicos asociados, como un (cada vez menos teléfono) móvil convertido en verdadera prótesis digital de nuestras capacidades intelectuales.

La vertiente más engañosa de este fenómeno transformador es precisamente la que lo ha popularizado, es decir la cuantitativa

\section{Una herramienta conceptual, un escenario complejo}

El modelo descrito se estructura por tanto alrededor de tres elementos básicos (infotecnologías, personas y contenidos) y se articula sobre tres ejes principales (información, rela- ción y comunicación). Este modelo puede servir para explicar por ejemplo, cómo el fenómeno incipiente de las redes sociales se apoya aún en la transformación de personas en contenidos -como nos ilustra el tratamiento automatizado de los perfiles y patrones de uso en esas plataformas para los recomendadores sociales utilizados en el comercio electrónico-. O cómo la hipermultifuncionalidad de los dispositivos móviles modernos se apoya en la incorporación de capacidades intelectivas generalmente asociadas a la inteligencia humana.

De la misma manera, aun evitando la complejidad de otros instrumentos conceptuales que han servido para concebirlo, como es el caso particular del NET y el marco tecnocultural en el que se apoya (Sáez-Vacas, 2011), el modelo quiere servir como herramienta para el lector interesado en profundizar en cualquiera de esas dimensiones, incorporando la íntima relación de las mismas en un marco conceptual que le ayude a explicar una realidad socioeconómica en plena transformación, afectada a todos los niveles por un desarrollo tecnológico sin precedentes.

Un ejemplo de aplicación aparece representado sintéticamente en la figura 1. "Escuchar, enlazar, compartir..., ¿influir?" son las acciones que identificaba bajo la lógica de este modelo para ilustrar la dinámica popularizada por los medios sociales.

Es importante que el lector pueda hacer este tipo de ejercicios en otros ámbitos, como pueden ser los directamente relacionados con su actividad profesional. Las funcionalidades que nos ofrecen los instrumentos técnicos de la Red para el filtraje de contenidos, su edición, publicación y su utilización en diferentes entornos, siguen apoyándose en una infraestructura reticular y en la capacidad básica para "enlazar" recursos hipertextuales, conectando los nodos de una Red, que por definición es universal y digital (Sáez-Vacas, 2004).

\section{Bibliografía}

Cohen, Sarah; Hamilton, James; Turner, Fred. "Computational journalism. How computer scientists can empower journalists, democracy's watchdogs, in the production of news in the public interest". Communications of the ACM, October 2011, v. 54, n. 10, pp. 66-71.

http://cacm.acm.org/magazines/2011/10/131400-compu tational-journalism/fulltext

http://dx.doi.org/10.1145/2001269.2001288

Hendler, James; Shadbolt, Nigel; Hall, Wendy; Berners-Lee, Tim; Weitzner, Daniel. "Web science: an interdisciplinary approach to understanding the web". Communications of the ACM, July 2008, v. 51, n. 7, pp. 60-69.

http://dl.acm.org/citation.cfm?id=1364798

http://dx.doi.org/10.1145/1364782.1364798

Huff, Darrel. How to lie with statistics. New York: Norton, 1954. ISBN 9780393310728

Illich, Ivan. Tools for conviviality. Editorial Harper \& Row, 1973. ISBN 9780060121389

http://clevercycles.com/tools_for_conviviality

Sáez-Vacas, Fernando. Más allá de internet: la Red universal 
digital. Madrid: Editorial Centro de Estudios Ramón Areces, 2004. ISBN 8480046295

Sáez-Vacas, Fernando. Cultura y tecnología en el nuevo entorno tecnosocial. Madrid: Editorial Fundación Rogelio Segovia para el Desarrollo de las Telecomunicaciones, 2011. ISBN 9788474023763.

http://www.gsi.dit.upm.es/ fsaez/intl/cultura_y_tecnolo gia.pdf

Sáinz-Peña, Rosa-María (coord.). Real time web: una nueva conciencia global. Madrid: Editorial Ariel, 2011. Colección Fundación Telefónica.

http://www.fundacion.telefonica.com/es/debateyconoci miento/media/publicaciones/Real_time_web_nw.pdf
The cocktail analysis. Tercera oleada del Observatorio de Redes Sociales, enero 2011.

http://www.tcanalysis.com/2011/02/22/publicamos-la3\%C2\%AA-ola-del-observatorio-de-redes-sociales http://redes-sociales.the-cocktail.com

Van-Bellenghem, Steven (ed.). Social media around the world, 2011. InSites Consulting.

http://www.slideshare.net/stevenvanbelleghem/socialmedia-around-the-world-2011

Van-Ark, Bart (ed.). Un mundo conectado: las TIC transforman sociedades, culturas y economías. Madrid: Editorial Ariel, 2011. Colección Fundación Telefónica. ISBN 9788408103295 online information 2011

29 Nov - 1 Dec

Olympia Conference Centre, London
El profesional de la información

\title{
Spanish Meeting Point
}

\author{
Stand 376, Conferencia Online Information \\ Londres, 29 noviembre al 1 de diciembre 2011
}

Un año más, la revista "El profesional de la información" organiza el stand "Spanish Meeting Point" en la conferencia Online Information de Londres. http://www.online-information.co.uk

Este stand sirve de punto de encuentro para los profesionales de la información hispanohablantes. En éste habrá actividades varias.

La entrada a la Feria es gratuita -si uno se inscribe online antes de llegar.

La financiación de este stand ha sido posible gracias a:

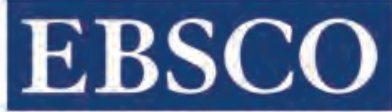

INFORMATION SERVICES

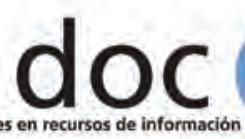

consultores en recursos de información

스 Springer

the language of science 


\section{Rapidez, precisión, flexibilidad}

En el siglo XXI, más que nunca, la información es poder. Ayudar a empresas e instituciones a gestionar y rentabilizar su caudal informativo, tanto el que poseen como el que genera diariamente su actividad, es el objetivo y la razón de ser de Baratz.

www.baratz.es 